\title{
An Efficient Isocyanide-Based Three-Component Synthesis of Novel Ketenimines
}

\author{
Hamideh Emtiazi and Mohammad Ali Amrollahi*
}

Department of Chemistry, College of Science, Yazd University, Yazd. Iran, P.O.Box 89195-741

${ }^{*}$ Corresponding author: mamrollahi@yazd.ac.ir

\section{ABSTRACT}

This study provides a description of an efficient and simple procedure for the synthesis of dimethyl 2-(9-aryl)-3,3,6,6tetramethyl-1,8-diox-1,2,3,4,5,6,7,8-octahydroacridin-10(9H)-yl)-3-((cyclohexylimino)methylene)succinate via a one-pot three-component reaction of cyclohexyl isocyanide, dimethyl acetylenedicarboxylate and hexahydroacridine-1,8(2H,5H)diones in $\mathrm{CH}_{2} \mathrm{Cl}_{2}$ at room temperature. Short reaction times, good to high yields and the novelty are the remarkable advantages of this work.

Keywords: Hexahydroacridine; acetylenedicarboxylate; isocyanide; one-pot

\section{Council for Innovative Research}

Peer Review Research Publishing System

Journal: Journal of Advances in Chemistry

Vol. 10, No. 3

editorjaconline@gmail.com

www.cirjac.com 


\section{INTRODUCTION}

Multicomponent reactions (MCRs) are special type of synthetically useful organic reactions in which three or more different starting materials react to give a final product in a one-pot procedure [1-3]. MCRs have drawn high efforts in recent years, because they increase the efficiency by combining several operational steps without isolating the intermediates or changing the reaction conditions. This reduces the reaction time and saves both energy and raw materials, promoting the green chemistry [4-6]. Isocyanide-based multicomonent reactions (IMCRs) are especially important in this area due to the adventages that they offer to the field of combinatorial chemistry $[7,8]$. Ketenimine derivatives are reactive synthetic intermediates, which react readily with a wide range of nucleophiles, electrophiles or radicals to afford the corresponding nitrogen-containing heterocycles [9, 10]. They also undergo many pericyclic reactions such as electrocyclic ring closures, [2+2] and [4+2] cycloaddition reactions [11-13]. Ketenimine derivatives have been prepared via various procedures such as imidation of ketene precursors [14], dehydrohalogenation of imidoyl halides under basic conditions [15], treatment of nitriles with a Brønsted base followed by substitution reaction [16], and the reaction of isocyanides, acetylenic esters, and various compounds as proton source [17-21]. Herein, we report synthesis of novel ketenimines via a one-pot threecomponent reaction of cyclohexyl isocyanide, dimethyl acethylendicarboxylate and hexahydroacridine-1,8(2H,5H)-dione in $\mathrm{CH}_{2} \mathrm{Cl}_{2}$ at room temperature.

\section{EXPERIMENTAL}

Products were characterized by FT-IR, ${ }^{1} \mathrm{H}$-, and ${ }^{13} \mathrm{C}-\mathrm{NMR}$ spectra. FT-IR spectra were run on a Bruker, Eqinox 55 spectrometer. ${ }^{1} \mathrm{H}$-, and ${ }^{13} \mathrm{C}-\mathrm{NMR}$ spectra were obtained using Bruker Avance $400 \mathrm{MHz}$ spectrometers (DRX). Melting points were determined by a Büchi melting point B-540 B.V.CHI apparatus. Elemental analyses were performed using a Costech ECS $4010 \mathrm{CHN}$ analyzer. Column chromatography was performed on silica gel (230-400) mesh. Analytical TLC was performed on pre-coated plastic sheets of silica gel G/UV-254 of $0.2 \mathrm{~mm}$ thickness.

\section{General procedure for the synthesis of hexahydroacridinedione derivatives (3a-j, Table 2).}

A mixture of an aldehyde $(1 \mathrm{mmol})$, dimedone $(2 \mathrm{mmol}, 0.280 \mathrm{~g})$, ammonium acetate $(1.2 \mathrm{mmol}, 0.092 \mathrm{~g})$ and $\mathrm{Mg}(\mathrm{ClO})_{2}$ $8 \mathrm{H}_{2} \mathrm{O}(0.025 \mathrm{~g})$ was stirred under solvent-free condition at $80^{\circ} \mathrm{C}$ for $30 \mathrm{~min}$. After completion of the reaction, for isolation of catalyst, the mixture was dissolved in hot $\mathrm{CHCl}_{3}$ and filtered. The solvent of the resulted filtrate was evaporated and the pure product was obtained by recrystalization from ethanol.

\section{Typical procedure for the synthesis of ketenimine derivatives (4a-j, Table 2).}

To a magnetically stirred solution of 3,3,6,6-tetramethyl-9-phenyl-3,4,6,7,9,10-hexahydroacridine-1,8(2H,5H)-dione (1 $\mathrm{mmol})$ and dimethyl acetylenedicarboxylate $(1 \mathrm{mmol})$ in dry $\mathrm{CH}_{2} \mathrm{Cl}_{2}(3 \mathrm{~mL})$ was added a solution of cyclohexyl isocyanide $(1 \mathrm{mmol})$ in dry $\mathrm{CH}_{2} \mathrm{Cl}_{2}(2 \mathrm{~mL})$ dropwise at room temperature over $10 \mathrm{~min}$ and the mixture was stirred at room temperature for $4 \mathrm{~h}$. After completion of the reaction as indicated by TLC, the solvent was removed under reduced pressure, and dimethyl 2-((cyclohexylimino)methylene)-3-(3,3,6,6-tetramethyl-1,8-dioxo-9-phenyl-1,2,3,4,5,6,7,8-octahydroacridin$10(9 \mathrm{H})$-yl)succinate $(\mathbf{4 a}$, Table 2 ) was separated by silica gel column chromatography using a hexane/ethyl acetate (70:30) as eluent.

\section{Dimethyl-2-((cyclohexylimino)methylene)-3-(3,3,6,6-tetramethyl-9-(3-nitrophenyl)-1,8-dioxo- 1,2,3,4,5,6,7,8-octahydroacridin-10(9H)-yl)succinate (4b; Table 2, Entry 2):}

Yellow solid; $\mathrm{mp} 189-190^{\circ} \mathrm{C}$. IR: $\mathrm{v}_{\max }=2934,2858,2056,1744,1694,1635,1577,1528,1436,1349,1220,732 \mathrm{~cm}^{-1}$. ${ }^{1} \mathrm{H}-\mathrm{NMR}(400 \mathrm{MHz}$, DMSO-d $\mathrm{d}): \delta=0.95(\mathrm{~s}, 3 \mathrm{H}), 0.97(\mathrm{~s}, 3 \mathrm{H}), 1.00(\mathrm{~s}, 3 \mathrm{H}), 1.03(\mathrm{~s}, 3 \mathrm{H}), 1.07-1.76(\mathrm{~m}, 10 \mathrm{H}), 2.14-2.25$ (2d, $J=16.0 \mathrm{~Hz}, 4 \mathrm{H}), 2.31(\mathrm{~d}, J=16.4 \mathrm{~Hz}, 2 \mathrm{H}), 2.43(\mathrm{~d}, J=16.0 \mathrm{~Hz}, 2 \mathrm{H}), 3.60(\mathrm{~s}, 3 \mathrm{H}), 3.76(\mathrm{~m}, 1 \mathrm{H}), 3.81(\mathrm{~s}, 3 \mathrm{H}), 5.28(\mathrm{~s}$, $1 \mathrm{H}), 5.74(\mathrm{~s}, 1 \mathrm{H}), 7.22(\mathrm{t}, J=7.6 \mathrm{~Hz}, 1 \mathrm{H}), 7.65(\mathrm{~d}, J=8.0 \mathrm{~Hz}, 1 \mathrm{H}), 7.83(\mathrm{~d}, J=8.4 \mathrm{~Hz}, 1 \mathrm{H}), 7.90(\mathrm{~s}, 1 \mathrm{H}) .{ }^{13} \mathrm{C}-\mathrm{NMR}(100$ MHz, DMSO-d $\left.\mathrm{d}_{6}\right) \delta=24.0,25.1,26.4,27.3,29.2,32.3,33.5,40.1,40.9,49.7,50.4,52.1,53.5,57.1,61.1,114.9,116.1$, 121.1, 122.5, 128.5, 135.2, 147.2, 151.7, 152.6, 169.1, 172.0, 196.0. Anal. calc. for $\mathrm{C}_{36} \mathrm{H}_{43} \mathrm{~N}_{3} \mathrm{O}_{8}(643.31): \mathrm{C} 66.96, \mathrm{H} 6.71$, N 6.51; found: C 66.6, H 6.5, N 6.8.

\section{Dimethyl-2-((cyclohexylimino)methylene)-3-(9-(4-fluorophenyl)-3,3,6,6-tetramethyl-1,8-dioxo- 1,2,3,4,5,6,7,8-octahydroacridin-10(9H)-yl)succinate (4c; Table 2, Entry 3):}

Yellow solid; $\mathrm{mp} 161-162{ }^{\circ} \mathrm{C}$. IR: $\mathrm{v}_{\max }=2932,2079,1742,1692,1649,1435,1219 \mathrm{~cm}^{-1}$. ${ }^{1} \mathrm{H}-\mathrm{NMR}\left(400 \mathrm{MHz}, \mathrm{DMSO}-\mathrm{d}_{6}\right): \delta$ $=1.00(\mathrm{~s}, 3 \mathrm{H}), 1.04(\mathrm{~s}, 3 \mathrm{H}), 1.08(\mathrm{~s}, 3 \mathrm{H}), 1.13(\mathrm{~s}, 3 \mathrm{H}), 1.18-1.82(\mathrm{~m}, 10 \mathrm{H}), 2.38(\mathrm{~d}, J=16.0 \mathrm{~Hz}, 2 \mathrm{H}), 2.44(\mathrm{~d}, J=16.0 \mathrm{~Hz}$, 2H), 2.56-2.62 (2d, J=16.0 Hz, 4H), $3.72(\mathrm{~s}, 3 \mathrm{H}), 3.85(\mathrm{~m}, 1 \mathrm{H}), 3.89(\mathrm{~s}, 3 \mathrm{H}), 5.25(\mathrm{~s}, 1 \mathrm{H}), 5.81(\mathrm{~s}, 1 \mathrm{H}), 6.79-6.83(\mathrm{~m}, 2 \mathrm{H})$, 7.18-7.21 (m, 2H). ${ }^{13} \mathrm{C}-\mathrm{NMR}\left(100 \mathrm{MHz}\right.$, DMSO-d $\left.\mathrm{d}_{6}\right) \delta=23.0,24.6,25.1,26.3,29.2,30.9,33.1,40.0,40.8,49.9,50.56$, $52.1,53.5,56.9,63.9,114.5,116.5,129.3,130.9,145.8,152.2,156.7,163.5,165.3,196.0$. Anal. calc. for $\mathrm{C}_{36} \mathrm{H}_{43} \mathrm{FN}_{2} \mathrm{O}_{6}$ (618.31): C 69.88, H 7.00, N 4.53; found: C 71.2, H 7.1, N 4.4.

Dimethyl-2-(9-(4-chlorophenyl)-3,3,6,6-tetramethyl-1,8-dioxo-1,2,3,4,5,6,7,8-octahydroacridin-10(9H)-yl)3-((cyclohexylimino)methylene)succinate (4d; Table 2, Entry 4):

Yellow solid; $\mathrm{mp} 171-173^{\circ} \mathrm{C}$. IR: $\mathrm{v}_{\max }=2930,2074,1737,1693,1629,1574,1435,1362,1217,1014,851 \mathrm{~cm}^{-1} .{ }^{1} \mathrm{H}-\mathrm{NMR}$ $\left(400 \mathrm{MHz}, \mathrm{DMSO}-\mathrm{d}_{6}\right): \delta=1.00(\mathrm{~s}, 3 \mathrm{H}), 1.04(\mathrm{~s}, 3 \mathrm{H}), 1.08(\mathrm{~s}, 3 \mathrm{H}), 1.13(\mathrm{~s}, 3 \mathrm{H}), 1.17-1.84(\mathrm{~m}, 10 \mathrm{H}), 2.22-2.34(2 \mathrm{~d}, J=17.2$ $\mathrm{Hz}, 4 \mathrm{H}), 2.36-2.45(\mathrm{~d}, J=16.0 \mathrm{~Hz}, 4 \mathrm{H}), 3.72(\mathrm{~s}, 3 \mathrm{H}), 3.85(\mathrm{~m}, 1 \mathrm{H}), 3.89(\mathrm{~s}, 3 \mathrm{H}), 5.24(\mathrm{~s}, 1 \mathrm{H}), 5.80(\mathrm{~s}, 1 \mathrm{H}), 7.16(\mathrm{~d}, J=8.8$ $\mathrm{Hz}, 2 \mathrm{H}), 7.83(\mathrm{~d}, \mathrm{~J}=8.4 \mathrm{~Hz}, 2 \mathrm{H}) .{ }^{13} \mathrm{C}-\mathrm{NMR}\left(100 \mathrm{MHz}\right.$, DMSO-d $\mathrm{d}_{6} \delta=24.0,25.1,26.3,27.2,29.3,31.2,33.1,40.0,40.9$, $49.8,50.5,52.1,53.5,56.9,64.4,115.5,117.1,127.9,131.3,143.5,151.1,152.3,164.1,169.8$, 195.9. Anal. calc. for 
$\mathrm{C}_{36} \mathrm{H}_{43} \mathrm{CIN}_{2} \mathrm{O}_{6}$ (634.28): C 68.07, H 6.82, N 4.41; found: C 67.7, H 7.1, N 4.5.

Dimethyl-2-(9-(3-bromophenyl)-3,3,6,6-tetramethyl-1,8-dioxo-1,2,3,4,5,6,7,8-octahydroacridin-10(9H)yl)-3-((cyclohexylimino)methylene)succinate (4e; Table 2, Entry 5):

Yellow solid; $\mathrm{mp} 185-187^{\circ} \mathrm{C}$. IR: $\mathrm{v}_{\max }=2932,2856,2076,1742,1681,1632,1470,1363,1220 \mathrm{~cm}^{-1} .{ }^{1} \mathrm{H}-\mathrm{NMR}(400 \mathrm{MHz}$ DMSO- $\left.\mathrm{d}_{6}\right): \delta=0.90(\mathrm{~s}, 3 \mathrm{H}), 0.95(\mathrm{~s}, 3 \mathrm{H}), 0.99(\mathrm{~s}, 3 \mathrm{H}), 1.04(\mathrm{~s}, 3 \mathrm{H}), 1.08-1.98(\mathrm{~m}, 10 \mathrm{H}), 2.24(\mathrm{~d}, J=16.0 \mathrm{~Hz}, 2 \mathrm{H}), 2.30(\mathrm{~d}$, $J=16.0 \mathrm{~Hz}, 2 \mathrm{H}), 2.39(\mathrm{~d}, J=16.4 \mathrm{~Hz}, 2 \mathrm{H}), 2.51(\mathrm{~d}, J=16.0 \mathrm{~Hz}, 2 \mathrm{H}), 3.65(\mathrm{~s}, 3 \mathrm{H}), 3.75(\mathrm{~m}, 1 \mathrm{H}), 3.80(\mathrm{~s}, 3 \mathrm{H}), 5.23(\mathrm{~s}, 1 \mathrm{H})$, $5.72(\mathrm{~s}, 1 \mathrm{H}), 6.90(\mathrm{t}, J=8.0 \mathrm{~Hz}, 1 \mathrm{H}), 7.60(\mathrm{~d}, J=7.6 \mathrm{~Hz}, 2 \mathrm{H}), 7.28(\mathrm{br}, 1 \mathrm{H}) .{ }^{13} \mathrm{C}-\mathrm{NMR}\left(100 \mathrm{MHz}, \mathrm{DMSO}-\mathrm{d}_{6}\right) \delta=22.7,25.2$, 26.6, 27.3, 29.0, 29.7, 30.2, 31.4, 33.2, 40.9, 49.8, 50.5, 53.6, 57.0, 61.1, 116.6, 119.1, 122.1, 126.8, 129.3, 130.9, 147.3, 151.3, 152.3, 165.8, 168.8, 196.0. Anal. calc. for $\mathrm{C}_{36} \mathrm{H}_{43} \mathrm{BrN}_{2} \mathrm{O}_{6}$ (678.23): C 63.62, H 6.38, N 4.12; found: C 63.8, H 6.5, N 3.9 .

Dimethyl-2-((cyclohexylimino)methylene)-3-(3,3,6,6-tetramethyl-1,8-dioxo-9-(p-tolyl)-1,2,3,4,5,6,7,8octahydroacridin-10(9H)-yl)succinate (4f; Table 2, Entry 6):

Yellow solid; $\mathrm{mp} 165-167^{\circ} \mathrm{C}$. IR: $\mathrm{v}_{\max }=2927,2854,2077,1741,1696,1634,1588,1509,1437,1363,1221,762 \mathrm{~cm}^{-1}$. ${ }^{1} \mathrm{H}-\mathrm{NMR}\left(400 \mathrm{MHz}, \mathrm{DMSO}-\mathrm{d}_{6}\right): \delta=0.93(\mathrm{~s}, 3 \mathrm{H}), 0.94(\mathrm{~s}, 3 \mathrm{H}), 0.98(\mathrm{~s}, 3 \mathrm{H}), 1.03(\mathrm{~s}, 3 \mathrm{H}), 1.12-1.76(\mathrm{~m}, 10 \mathrm{H}), 2.12(\mathrm{~s}, 3 \mathrm{H})$, 2.12-2.24 (2d, $J=16.0 \mathrm{~Hz}, 4 \mathrm{H}), 2.28(\mathrm{~d}, J=16.0 \mathrm{~Hz}, 2 \mathrm{H}), 2.32(\mathrm{~d}, J=16.4 \mathrm{~Hz}, 2 \mathrm{H}), 3.63(\mathrm{~s}, 3 \mathrm{H}), 3.80(\mathrm{~s}, 3 \mathrm{H}), 3.89(\mathrm{~m}$, $1 \mathrm{H}), 5.14(\mathrm{~s}, 1 \mathrm{H}), 5.72(\mathrm{~s}, 1 \mathrm{H}), 6.83(\mathrm{~d}, \mathrm{~J}=8.0 \mathrm{~Hz}, 2 \mathrm{H}), 7.03(\mathrm{~d}, \mathrm{~J}=8.0 \mathrm{~Hz}, 2 \mathrm{H}) .{ }^{13} \mathrm{C}-\mathrm{NMR}\left(100 \mathrm{MHz}, \mathrm{DMSO}-\mathrm{d}_{6}\right) \delta=21.0$ 24.0, 25.1, 26.5, 27.3, 29.7, 31.2, 33.2, 40.8, 42.3, 49.9, 50.6, 52.1, 53.5, 56.8, 63.8, 117.5, 127.7, 128.5, 134.9, 142.0, 150.8, 151.8, 167.8, 170.0, 196.2. Anal. calc. for $\mathrm{C}_{37} \mathrm{H}_{46} \mathrm{~N}_{2} \mathrm{O}_{6}(614.34)$ : C 72.29, H 7.54, N 4.56; found: C 71.9, $\mathrm{H} 7.5, \mathrm{~N}$ 4.9 .

Dimethyl-2-((cyclohexylimino)methylene)-3-(9-(4-hydroxy-3-methoxyphenyl)-3,3,6,6-tetramethyl-1,8dioxo-1,2,3,4,5,6,7,8-octahydro acridin-10(9H)-yl)succinate (4g; Table 2, Entry 7):

Yellow solid; $\mathrm{mp} 160-162{ }^{\circ} \mathrm{C}$. IR: $\mathrm{v}_{\max }=3411,2926,2075,1742,1630,1451,1364,1220 \mathrm{~cm}^{-1} .{ }^{1} \mathrm{H}-\mathrm{NMR}(400 \mathrm{MHz}$, DMSO-d $\left.\mathrm{d}_{6}\right): \delta=0.87(\mathrm{~s}, 3 \mathrm{H}), 0.95(\mathrm{~s}, 3 \mathrm{H}), 0.99(\mathrm{~s}, 3 \mathrm{H}), 1.04(\mathrm{~s}, 3 \mathrm{H}), 1.09-1.75(\mathrm{~m}, 10 \mathrm{H}), 2.10-2.14(\mathrm{~m}, 4 \mathrm{H}), 2.29(\mathrm{~d}, J=$ $16.0 \mathrm{~Hz}, 2 \mathrm{H}), 2.36(\mathrm{~d}, J=16.4 \mathrm{~Hz}, 2 \mathrm{H}), 3.60(\mathrm{~s}, 3 \mathrm{H}), 3.75(\mathrm{~m}, 1 \mathrm{H}), 3.88(\mathrm{~s}, 3 \mathrm{H}), 3.95(\mathrm{~s}, 3 \mathrm{H}), 5.11(\mathrm{~s}, 1 \mathrm{H}), 5.72(\mathrm{~s}, 1 \mathrm{H}), 6.38$ $(\mathrm{dd}, J=8.0 \mathrm{~Hz}, J=1.6 \mathrm{~Hz}, 1 \mathrm{H}), 6.53(\mathrm{~d}, J=8.0 \mathrm{~Hz}, 1 \mathrm{H}), 6.96(\mathrm{~d}, J=2.0 \mathrm{~Hz}, 1 \mathrm{H}) .{ }^{13} \mathrm{C}-\mathrm{NMR}\left(100 \mathrm{MHz}, \mathrm{DMSO}-\mathrm{d}_{6}\right) \delta=$ 22.7, 24.0, 25.1, 26.4, 27.4, 29.7, 30.9, 31.5, 33.1, 40.8, 49.9, 52.2, 53.5, 55.9, 56.9, 64.1, 113.5, 115.9, 119.3, 130.9, 137.2, 143.56, 145.7, 150.9, 152.0, 163.7, 169.9, 196.2. Anal. calc. for $\mathrm{C}_{37} \mathrm{H}_{46} \mathrm{~N}_{2} \mathrm{O}_{8}$ (646.33): C 68.71, $\mathrm{H} 7.71, \mathrm{~N} 4.33$; found: C 68.6, H 7.5, N 4.6.

Dimethyl-2-((cyclohexylimino)methylene)-3-(9-(4-methoxyphenyl)-3,3,6,6-tetramethyl-1,8-dioxo1,2,3,4,5,6,7,8-octahydroacridin-10(9H)-yl)succinate (4h; Table 2, Entry 8):

Yellow solid; $\mathrm{mp} 159-160{ }^{\circ} \mathrm{C}$. IR: $\mathrm{v}_{\max }=2930,2855,2075,1742,1696,1633,1577,1508,1437,1363,1263,1220,874$ $\mathrm{cm}^{-1} .{ }^{1} \mathrm{H}-\mathrm{NMR}\left(400 \mathrm{MHz}, \mathrm{DMSO}_{-} \mathrm{d}_{6}\right): \delta=0.82(\mathrm{~s}, 3 \mathrm{H}), 0.95(\mathrm{~s}, 3 \mathrm{H}), 0.99(\mathrm{~s}, 3 \mathrm{H}), 1.04(\mathrm{~s}, 3 \mathrm{H}), 1.12-1.78(\mathrm{~m}, 10 \mathrm{H}), 2.07$ $(\mathrm{d}, J=16.8 \mathrm{~Hz}, 2 \mathrm{H}), 2.15(\mathrm{~d}, J=16.4 \mathrm{~Hz}, 2 \mathrm{H}), 2.20-2.28(2 \mathrm{~d}, J=17.2 \mathrm{~Hz}, 4 \mathrm{H}), 3.67(\mathrm{~s}, 3 \mathrm{H}), 3.75(\mathrm{~s}, 3 \mathrm{H}), 3.80(\mathrm{~m}, 3 \mathrm{H})$, $3.88(\mathrm{~s}, 1 \mathrm{H}), 5.08(\mathrm{~s}, 1 \mathrm{H}), 5.98(\mathrm{~s}, 1 \mathrm{H}), 6.68(\mathrm{~d}, J=8.4 \mathrm{~Hz}, 2 \mathrm{H}), 7.21(\mathrm{~d}, J=8.4 \mathrm{~Hz}, 2 \mathrm{H}) .{ }^{13} \mathrm{C}-\mathrm{NMR}(100 \mathrm{MHz}$, DMSO-d 6$) \delta$ $=23.7,25.3,26.6,29.4,29.7,31.4,32.0,32.4,33.0,39.4,50.5,52.8,52.9,53.6,55.2,63.7,115.1,123.9,129.9,138.0$, 145.9, 147.6, 157.9, 164.2, 165.0, 195.5. Anal. calc. for $\mathrm{C}_{37} \mathrm{H}_{46} \mathrm{~N}_{2} \mathrm{O}_{7}(630.33)$ : C 70.45, H 7.35, N 4.44; found: C 70.7, $\mathrm{H}$ 7.2, N 4.7 .

Dimethyl-2-((cyclohexylimino)methylene)-3-(9-(3,4-dimethoxyphenyl)-3,3,6,6-tetramethyl-1,8-dioxo1,2,3,4,5,6,7,8-octahydroacridin-10(9H)-yl)succinate (4i; Table 2, Entry 9):

Yellow solid; $\mathrm{mp} 177-179{ }^{\circ} \mathrm{C}$. IR: $\mathrm{v}_{\max }=2934,2070,1744,1701,1632,1439,1221 \mathrm{~cm}^{-1}$. ${ }^{1} \mathrm{H}-\mathrm{NMR}\left(400 \mathrm{MHz}, \mathrm{DMSO}-\mathrm{d}_{6}\right): \delta$ $=1.04(\mathrm{~s}, 3 \mathrm{H}), 1.06(\mathrm{~s}, 3 \mathrm{H}), 1.08(\mathrm{~s}, 3 \mathrm{H}), 1.13(\mathrm{~s}, 3 \mathrm{H}), 1.27-1.83(\mathrm{~m}, 10 \mathrm{H}), 2.24-2.47(\mathrm{~m}, 8 \mathrm{H}), 3.66(\mathrm{~s}, 3 \mathrm{H}), 3.77(\mathrm{~s}, 3 \mathrm{H})$, $3.84(\mathrm{~s}, 3 \mathrm{H}), 3.88(\mathrm{~s}, 3 \mathrm{H}), 3.97(\mathrm{~m}, 1 \mathrm{H}), 5.22(\mathrm{~s}, 1 \mathrm{H}), 5.81(\mathrm{~s}, 1 \mathrm{H}), 6.61(\mathrm{~s}, 2 \mathrm{H}), 6.98(\mathrm{~s}, 1 \mathrm{H}) .{ }^{3} \mathrm{C}-\mathrm{NMR}(100 \mathrm{MHz}$, DMSO$\left.\mathrm{d}_{6}\right) \delta=24.0,25.1,26.4,27.5,29.4,29.7,30.7,33.2,40.1,40.8,49.9,50.6,52.0,53.5,55.9,56.8,64.1,112.2,115.8$, 117.3, 119.0, 137.9, 147.0, 148.3, 150.9, 152.0 166.7, 169.9, 196.2. Anal. calc. for $\mathrm{C}_{38} \mathrm{H}_{48} \mathrm{~N}_{2} \mathrm{O}_{8}(660.34)$ : C 69.07, H 7.32, N 4.24; found: C 68.9, H 7.0, N 4.5.

Dimethyl-2-((cyclohexylimino)methylene)-3-(9-(4-(dimethylamino)phenyl)-3,3,6,6-tetramethyl-1,8-dioxo1,2,3,4,5,6,7,8-octahydroacridin-10 (9H)-yl)succinate (4j; Table 2, Entry 10):

Yellow solid; $\mathrm{mp} 156-157^{\circ} \mathrm{C}$. IR: $\mathrm{v}_{\max }=3400,2983,1697,1665,1607,1488,1364,1175,1137,1058,897,767 \mathrm{~cm}^{-1}$. ${ }^{1} \mathrm{H}-$ NMR $\left(400 \mathrm{MHz}\right.$, DMSO-d $\left.\mathrm{d}_{6}\right): \delta=0.84(\mathrm{~s}, 6 \mathrm{H}), 0.96(\mathrm{~s}, 6 \mathrm{H}), 1.00-1.77(\mathrm{~m}, 10 \mathrm{H}), 2.04-2.27(\mathrm{~m}, 8 \mathrm{H}), 2.79(\mathrm{~s}, 6 \mathrm{H}), 3.66(\mathrm{~m}$, $1 \mathrm{H}), 3.75(\mathrm{~s}, 3 \mathrm{H}), 3.88(\mathrm{~s}, 3 \mathrm{H}), 5.05(\mathrm{~s}, 1 \mathrm{H}), 5.98(\mathrm{~s}, 1 \mathrm{H}), 6.52(\mathrm{~d}, \mathrm{~J}=8.8 \mathrm{~Hz}, 2 \mathrm{H}), 7.14(\mathrm{~d}, \mathrm{~J}=8.4 \mathrm{~Hz}, 2 \mathrm{H}) .{ }^{13} \mathrm{C}-\mathrm{NMR}(100$ $\mathrm{MHz}$, DMSO-d $\left.\mathrm{d}_{6}\right) \delta=23.7,25.3,26.8,29.0,29.8,32.0,37.1,38.5,40.8,49.6,52.8,52.9,54.9,56.1,59.2,63.5,115.3$, 123.9, 128.9, 134.2, 145.5, 147.8, 149.1, 164.3, 165.1,195.5. Anal. calc. for $\mathrm{C}_{38} \mathrm{H}_{49} \mathrm{~N}_{3} \mathrm{O}_{6}(643.36): \mathrm{C} 70.89, \mathrm{H} 7.67, \mathrm{~N} 6.53$; found: C 71.2, H 7.5, N 6.9. 


\section{RESULTS AND DISUSSTION}

The hexahydroacridindione derivatives were synthesized from the reaction of dimedone, aldehyde and ammonium acetate in the presence of $\mathrm{Mg}\left(\mathrm{ClO}_{4}\right)_{2} 8 \mathrm{H}_{2} \mathrm{O}$ (Scheme 1).

$$
\mathrm{R}: \mathrm{C}_{6} \mathrm{H}_{5}, 3-\mathrm{NO}_{2} \mathrm{C}_{6} \mathrm{H}_{4}, 4-\mathrm{FC}_{6} \mathrm{H}_{4}, 4-\mathrm{ClC}_{6} \mathrm{H}_{4}, 3-\mathrm{BrC}_{6} \mathrm{H}_{4}, 4-\mathrm{MeC}_{6} \mathrm{H}_{4}, 4-\mathrm{OH}-3-\mathrm{MeOC}_{6} \mathrm{H}_{3}, 4-\mathrm{MeOC}_{6} \mathrm{H}_{4}, 3,4-(\mathrm{MeO})_{2} \mathrm{C}_{6} \mathrm{H}_{3}, 4-\mathrm{N}_{(\mathrm{Me})_{2} \mathrm{C}_{6} \mathrm{H}_{4}}
$$

\section{Scheme 1}

Cyclohexyl isocyanide and dimethyl acetylenedicarboxylate in the presence of hexahydroacridinediones as $\mathrm{NH}$-acids undergo a smooth 1:1:1 addition reaction in $\mathrm{CH}_{2} \mathrm{Cl}_{2}$ at room temperature to produce ketenimine derivatives. The structures of the products were assigned on the basis of IR, ${ }^{1} \mathrm{H}$-, and ${ }^{13} \mathrm{C}-\mathrm{NMR}$. The IR spectra of $4 \mathrm{a}$ exhibited a strong absorption band for the ketenimine moiety at about $2083 \mathrm{~cm}^{-1}$ and for the carbonyl groups at 1745 and $1692 \mathrm{~cm}^{-1}$. The ${ }^{1} \mathrm{H}-\mathrm{NMR}$ spectrum of $\mathbf{4 a}$ exhibited four single sharp lines for four methyl groups of dimedone $(\delta=1.01,1.03,1.08,1.12)$, three multiplet for the five $\mathrm{CH}_{2}$ of cyclohexyl ring $(\delta=1.27-1.84)$, a multiplet for four $\mathrm{CH}_{2}$ of dimedone $(\delta=2.26-2.47)$, two singlet for two methyl groups in methoxy groups $(\delta=3.71,3.89)$, a multiplet for $\mathrm{N}-\mathrm{CH}$ cyclohexyl proton $(\delta=3.97)$, a singlet for Ph-CH proton $(\delta=5.28)$, and a singlet for $\mathrm{N}-\mathrm{CH}$ proton $(\delta=5.82)$ and two triplet and one doublet for five protons of phenyl ring $(\delta=7.03,7.11,7.22)$. The ${ }^{13} \mathrm{C}-\mathrm{NMR}$ spectrum of that ketenimine exhibited 25 sharp signals. ${ }^{1} \mathrm{H}-$ and ${ }^{13} \mathrm{C}-\mathrm{NMR}$ spectra of the crude mixture clearly indicate that the formation of the product leads to one diastereoisomer. Our attempts to detect the second diastereoisomer in the reaction mixture were not successful.

For optimizing the experimental conditions, the reaction between cyclohexyl isocyanide, dimethyl acethylendicarboxylate and 3,3,6,6-tetramethyl-9-phenyl-3,4,6,7,9,10-hexahydroacridine-1,8 $(2 \mathrm{H}, 5 \mathrm{H})$-dione was considered as a model reaction. To find the best solvent, several classic solvents were employed as media. The best solvent in terms of reaction yield and rate was found to be $\mathrm{CH}_{2} \mathrm{Cl}_{2}$ (Table 1).

Table 1. Optimizations of the reaction conditions for one-pot synthesis of ketenimines

\begin{tabular}{ccc}
\hline Entry & Solvent & Yield(\%) \\
\hline 1 & THF & 70 \\
2 & Acetone & 60 \\
3 & $\mathrm{CH}_{2} \mathrm{Cl}_{2}$ & 80 \\
4 & $\mathrm{MeCN}$ & 40 \\
5 & $\mathrm{EtOH}$ & 30 \\
\hline
\end{tabular}

Using these optimized reaction conditions, we extended our study to different hexahydroacridinediones to prepare a series of ketenimine derivatives (Table 2). For different substrates, the reaction could be completed in $4 \mathrm{~h}$ with high yields, with the substrates having either electron-donating groups or electron-withdrawing groups. 
Table 2. One-pot three-component synthesis of various ketenimines at room temperature

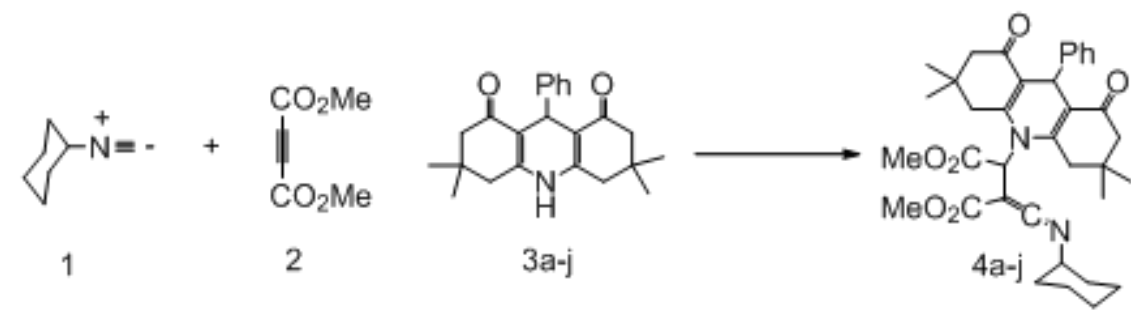

\begin{tabular}{clllll}
\hline Entry & $\mathrm{R}$ & 3 & 4 & Yield $(\%)$ & $\mathrm{Mp}\left({ }^{\circ} \mathrm{C}\right)$ \\
\hline 1 & $\mathrm{C}_{6} \mathrm{H}_{5}$ & $3 \mathrm{a}$ & $4 \mathrm{a}$ & 80 & $186-187$ \\
2 & $3-\mathrm{NO}_{2} \mathrm{C}_{6} \mathrm{H}_{4}$ & $3 \mathrm{~b}$ & $4 \mathrm{~b}$ & 84 & $189-190$ \\
3 & $4-\mathrm{FC}_{6} \mathrm{H}_{4}$ & $3 \mathrm{c}$ & $4 \mathrm{c}$ & 83 & $161-162$ \\
4 & $4-\mathrm{ClC}_{6} \mathrm{H}_{4}$ & $3 \mathrm{~d}$ & $4 \mathrm{~d}$ & 82 & $171-173$ \\
5 & $3-\mathrm{BrC}_{6} \mathrm{H}_{4}$ & $3 \mathrm{e}$ & $4 \mathrm{e}$ & 82 & $185-187$ \\
6 & $4-\mathrm{MeC}_{6} \mathrm{H}_{4}$ & $3 \mathrm{f}$ & $4 \mathrm{f}$ & 78 & $165-167$ \\
7 & $4-\mathrm{OH}-3-\mathrm{MeOC}_{6} \mathrm{H}_{3}$ & $3 \mathrm{~g}$ & $4 \mathrm{~g}$ & 75 & $160-162$ \\
8 & $4-\mathrm{MeOC}_{6} \mathrm{H}_{4}$ & $3 \mathrm{~h}$ & $4 \mathrm{~h}$ & 81 & $159-160$ \\
9 & $3,4-\mathrm{DiMeOC} \mathrm{H}_{3}$ & $3 \mathrm{i}$ & $4 \mathrm{i}$ & 90 & $177-179$ \\
10 & $4-\mathrm{N}, \mathrm{N}-\mathrm{dimethylC}_{6} \mathrm{H}_{4}$ & $3 \mathrm{j}$ & $4 \mathrm{j}$ & 89 & $156-157$ \\
\hline
\end{tabular}

Although the mechanism of the reaction has not yet been established experimentally, the formation of the product can be rationalized as outlined in the Scheme 2.

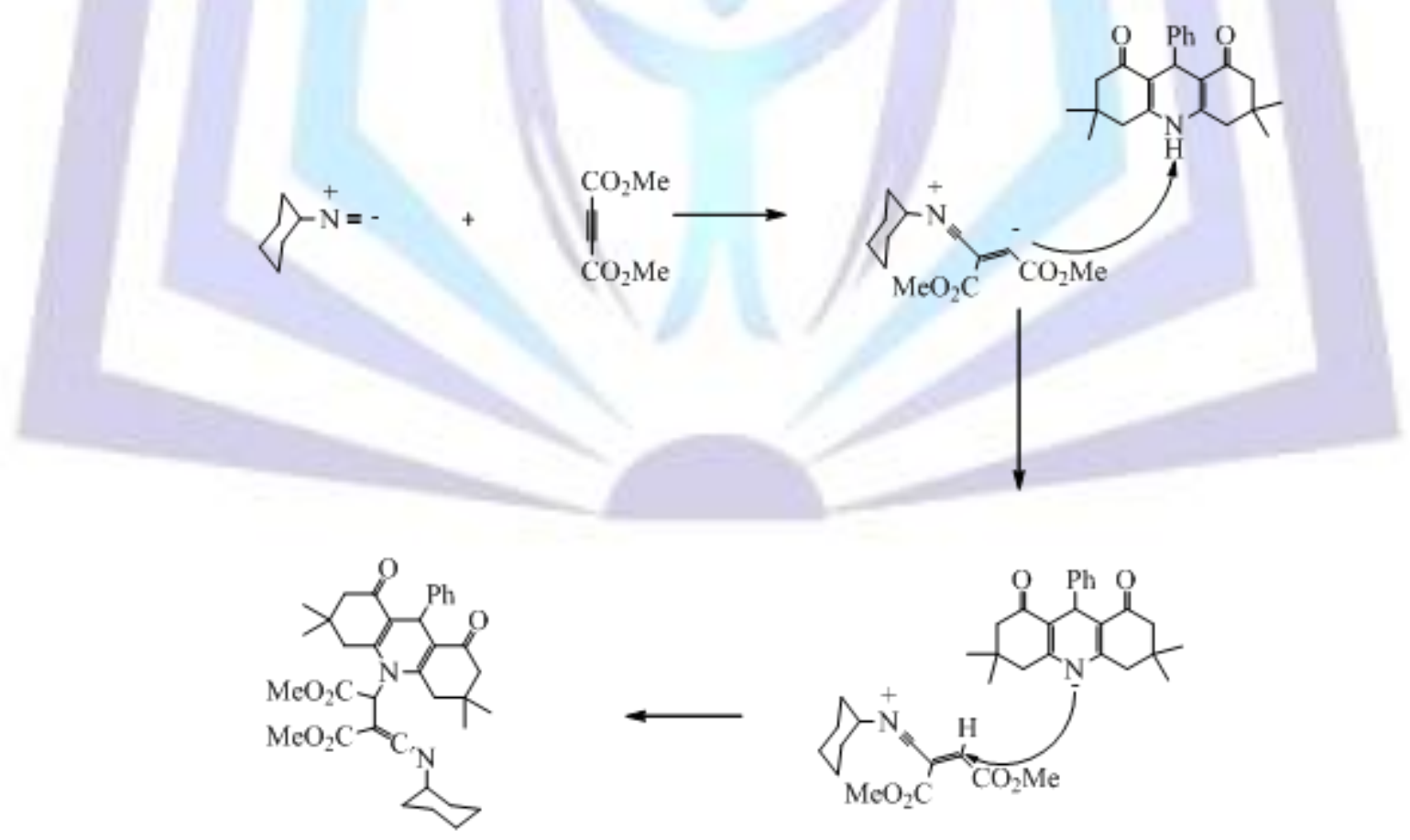

Scheme 2 


\section{ACKNOWLEDGMENTS}

The authors thank the Research Council of Yazd University for the financial support.

\section{REFERENCES}

[1] Shanthi, G., Perumal, P. T. 2009. InCl3-catalyzed efficient one-pot synthesis of 2-pyrrolo-3 '- yloxindoles. Tetrahydron Lett. 50, 3959-3962.

[2] Shirakawa, S., Kobayashi, S. 2006. Carboxylic acid catalyzed three-component aza-Friedel-Crafts reactions in water for the synthesis of 3-substituted indoles. Org. Lett. 8, 4939-4942.

[3] Zhang, H., Zhou, Z., Yao, Z., Xu, F., Shen, Q. 2009. Efficient synthesis of pyrimidinone derivatives by ytterbium chloride catalyzed Biginelli-type reaction under solvent-free conditions. Tetrahydron Lett. 50, 1622-1624.

[4] Heravi, M. M., Baghernejad, B., Oskooie, H. A. 2009. A novel three-component reaction for the synthesis of $\boldsymbol{N}$ cyclohexyl-3-aryl-quinoxaline-2-amines. Tetrahydron Lett. 50, 767-769.

[5] Huang, X., Zhang, T. 2009. Multicomponent reactions of pyridines, $\alpha$-bromo carbonyl compounds and silylaryl triflates as aryne precursors: a facile one-pot synthesis of pyrido[2,1-a]isoindoles. Tetrahydron Lett. 50, 208-211.

[6] Cui, S., Wang, J., Wang, Y. 2008. Copper-catalyzed multicomponent reaction: Facile access to novel phosphorus amidines. Org. Lett. 10, 1267-1269.

[7] Ugi I., Werner, B., Domling, A. 2003. The chemistry of isocyanides, their multiComponent reactions and their libraries. Molecules, 8, 53-56

[8] Esmaeili, A. A., Zangouei, M., Fakhari, A. R., Habibi, A. 2012. An efficient regioselective synthesis of highly functionalized 3-oxo-2,3-dihydro-5H-thiazolo[3,2-a]pyrimidines via an isocyanide-based three-component reaction. Tetrahydron Lett. 53, 1351-1353.

[9] Arrieta, A., Cossio, F. P., Lecea, B. 1999. Competitive mechanisms and origins of stereocontrol in the [2 +2 ] thermal cycloaddition between imines and keteniminium cations. A complementary entry to 2-azetidinones ( $\beta$-lactams) and related compounds. J. Org. Chem. 64, 1831-1842.

[10] Fromont, C., Masson, S. 1999. Reactivity of N-phenyl silylated ketenimines with electrophilic reagents. Tetrahedron 55, 5405-5418.

[11] Coffinier, D., Kaim, LE., Grimaud, L., Ruijter, E., Orru, RVA. 2011. A new multicomponent reaction for the synthesis of pyridines via cycloaddition of azadienes and ketenimines. Tetrahedron Lett. 52, 3023-325.

[12] Alajarin, M., Vidal, A., Tovar, F. 2005. The consecutive [2+2] cycloaddition-ring expansion route to diastereomeric 1,4diazepin-5-ones from imino-ketenimines. Alternative intramolecular transamidation of $\beta$-lactams. Tetrahedron 61 , 1531-1537.

[13] Molina, P., Vidal, A., Tovar, F. 1997. Electrocyclization of $\beta$-arylvinyl ketenimines: Formal syntheses of the alkaloid from marine origin, 5,8-dihydro7-methoxy-1,6-dimethylisoquinoline-5,8-dione, and 3-ethoxycarbonylrenierol synthesis. 8, 963-966.

[14] Staudinger, H., Hauuser, E. 1921. Über ketene, XXXVII. mitteilung. Keteniminderivate. Helv. Chim. Acta. 4, 887-896.

[15] Bestmann, H. J., Lienert, J., Mott, L. 1968. Reaktionen von triphenylphosphin sowie dessen hydrobromid und dibromid, IV1) reaktionen zwischen triphenylphosphin-dibromid und substituierten säureamiden. Ann. Chem. 718, 2432.

[16] Newman, M. S., Fukunaga, T., Miwa, T. 1960. Alkylation of nitriles: ketenimine formation. J. Am. Chem. Soc. 82, 873875.

[17] Yavari, I., Sanaeishoar, T., Azad, L., Ghazvini, M. 2011. Ketenimine N-functionalization of thiazolidine-2,4-diones with acetylenes and isocyanides. Mendeleev Commun. 21, 108-109.

[18] Baharfar, R., Jaafari, L., Azimi, R. 2011. Three-component reaction of alkyl isocyanides with acetylenic esters and pyridine-2-carboxaldoxime or $\alpha$-furildioxime: Synthesis and dynamic NMR study of ketenimines and bis(ketenimines). Chin. Chem. Lett 22, 943-946.

[19] Yavari, I., Nasiri, F., Djahaniani, H. 2004. Synthesis and dynamic NMR study of ketenimines derived from tert-butyl isocyanide, alkyl 2-arylamino-2-oxo-acetates, and dialkyl acetylenedicarboxylates. Mol. Divers. 8, 431-435.

[20] Anary-Abbasinejad, M., Moslemine, M. H., Anarak -Ardakani, H. 2009. One-pot synthesis of highly functionalized stable ketenimines of 2,2,2-trifluoro- $N$-aryl-acetamides. J. Fluorine Chem 130, 368-371.

[21] Adib, M., Sayahi, M. H., Behnam, B., Sheibani, E. 2006. Reaction between isocyanides and dialkyl acetylenedicarboxylates in the presence of hydantoins - A One-pot synthesis of stable ketenimines. Monatsh Chem. 137, $191-196$. 